\title{
Historical Introduction
}

\author{
K.C. Freeman \\ Mount Stromlo and Siding Spring Observatories, Private Bag, Weston \\ Creek PO, ACT 2611, Australia
}

\begin{abstract}
Our ideas about the surface brightness distribution of galaxies has changed greatly since 1970 . I contrast the view at that time with our present view of the subject, and then briefly discuss some topics in the studies of Low Surface Brightness Galaxies (LSBG) that I think are particularly interesting: the Tully-Fisher relation, LSBG as systems of high angular momentum, LSBG in clusters of galaxies, and the potential impact of the HIPASS survey.
\end{abstract}

\section{Introduction}

Around $1970 \mathrm{I}$ became interested in the exponential disk with surface brightness distribution $I(R)=I_{\circ} \exp (-\alpha R)$, assuming that it corresponded to an exponential surface density. I worked out the rotation curve for a self-gravitating exponential disk and was then curious about the range of values for the two parameters for the surface brightness distribution. I took the 36 disk galaxies with available surface photometry at the time. The data were mostly photographic, and the sample was inhomogeneous. Of the 36 galaxies, 28 seemed to have very similar blue central surface brightnesses $\mu_{B}(0)$ around $21.6 \mathrm{mag} \operatorname{arcsec}^{-2}$ after inclination correction for the path length through the galaxies. This was surprising, because much of the photometry was fairly rough. The sample also included a single low surface brightness galaxy, the Local Group member IC1613, with central surface brightness $\mu_{B}(0)=23.7$.

The conclusions of this work (Freeman 1970) are sometimes overstated. For example (from a recent paper)

In 1970 Freeman found that all spiral galaxies have a narrow distribution of central surface brightness centered around $\mu_{B}(0)=21.65 \pm 0.3$ mag $\operatorname{arcsec}^{-2}$.

This was all before dark matter in galaxies was well established. The apparently uniform central surface brightness for many of the galaxies, if interpreted as a uniform central surface density for the self-gravitating disks, led directly to a relationship between the angular momentum $J$ and mass $M$ of the disks of the form $J \propto M^{7 / 4}$. This seemed interesting for understanding the formation of disk galaxies, and several explanations for this relationship soon followed. This was the observational situation in 1970 . There was no attempt at this stage to correct for volume/visibility effects. 
The current situation is very different. Thanks to the work by Disney, Davies and Phillipps here in Cardiff, and to many who made the major photographic and CCD surveys over the last $\sim 15$ years, we now realise the importance of visibility effects and the existence of disk galaxies in significant numbers with much lower surface brightness. The present view of the true distribution of central surface brightnesses is summarised by Bothun et al. (1997: Fig. 1); the volume-corrected galaxy density per magnitude of surface brightness appears roughly flat from $\mu_{B}(0)=21.5$ down to at least 24 , although there remain differences of opinion about how to do this volume correction correctly. In any case, it seems clear that a significant fraction of disk galaxies have $\mu_{B}(0)>23$ mag $\operatorname{arcsec}^{-2}$.

Why is this important? The true distribution of surface brightnesses for disk galaxies affects our understanding of their formation processes. It is relevant to the distribution of the baryon to dark matter ratio for individual galaxies, and to the total baryon and dark matter contribution of galaxies in the universe.

\section{The Present View of Properties of LSBG}

(For references, please see the major reviews by Impey and Bothun (1997: IB) and Bothun et al. (1997: BIM).

- disks are mostly exponential.

- LSB does not necessarily mean low luminosity and low mass: see IB (Fig. 2) and BIM (Fig. 3). Giants with LSB disks exist, although they are rare and are usually detected optically by their high surface brightness (HSB) inner regions. Tables 1 and 2 give observational parameters and derived masses and $M / L$ ratios for four giant LSB galaxies (see also Freeman, 1997). Note their large scale lengths and their large $M / L$ values. In Table $1, V_{\circ}$ is the systemic velocity in $\mathrm{km} \mathrm{s}^{-1}, \mathrm{~W}_{\circ}$ is the corrected HI velocity width in $\mathrm{km} \mathrm{s}^{-1}$ and $\mathrm{h}$ is the scale length in $\mathrm{kpc}$. The quantities in Table 2 are in solar units.

- Galaxies cover most of the $\mu_{0}-\mathrm{h}$ plane for $\mu_{0}>20$ : the only apparently unpopulated region is for the high surface brightness systems with large scale lengths (see BIM Fig. 4).

- Colors and surface brightness are not related: fading is not the dominant reason for the LSB (see BIM Fig. 7).

- LSB disks are dark matter dominated at almost all radii (e.g. de Blok and McGaugh 1997). LSB halos tend to be less dense and have larger core radii; this may just be the consequence of the weaker baryonic compression of the halo by the lower surface brightness disk as it forms. The dark/luminous mass ratio is typically $>20$. This apparently high ratio may not be so extreme. The dark/luminous mass ratio is reliably known for only one HSBG (the Milky Way), for which it is again > 20 (e.g. Freeman 1996). 
Table 1: Dynamical and Structural Parameters for LSB Giants

\begin{tabular}{|l|rlrrr|}
\hline & $\mathrm{V}_{\mathrm{o}}$ & $\mathrm{B}(0)_{\mathrm{c}}$ & $\mathrm{W}_{\mathrm{o}}$ & $\mathrm{M}_{\mathrm{B}}$ & $\mathrm{h}$ \\
\hline Malin 1 & 24750 & 26 & 455 & -21.0 & 55 \\
F568-6 & 13830 & 23.4 & 674 & -21.2 & 16 \\
$1226+0105$ & 23655 & 23.3 & 405 & -21.6 & 12 \\
NGC 5084 & 1550 & & 700 & -20.8 & \\
\hline
\end{tabular}

Table 2: Masses and M/L Ratios for LSB Giants

\begin{tabular}{|l|ccl|}
\hline & $\mathrm{M}_{\text {tot }}$ & $\mathrm{M}_{\text {tot }} / \mathrm{L}$ & $\mathrm{M}_{\mathrm{H} \mathrm{I}}$ \\
\hline Malin 1 & $2 \times 10^{12}$ & 55 & $1 \times 10^{11}$ \\
F568-6 & $9 \times 10^{11}$ & 20 & $2 \times 10^{10}$ \\
$1226+0105$ & $2 \times 10^{12}$ & 30 & $2 \times 10^{10}$ \\
NGC 5084 & $1.3 \times 10^{12}$ & 45 & $9 \times 10^{9}$ \\
\hline
\end{tabular}

Table 3: Zero Surface Brightness Disks

\begin{tabular}{|l|cc|}
\hline & DDO 154 & NGC 2915 \\
\hline Stellar Mass & $5 \times 10^{7} M_{\odot}$ & $3 \times 10^{8} M_{\odot}$ \\
HI Mass & $2.7 \times 10^{8}$ & $1.3 \times 10^{9}$ \\
Total Mass & $3.8 \times 10^{9}$ & $2.7 \times 10^{10}$ \\
$M_{\text {total }} / M_{\text {baryon }}$ & 12 & 17 \\
\hline
\end{tabular}

\section{The Tully-Fisher Relation}

Zwaan et al. (1995) showed that most LSBG and HSBG follow the same TullyFisher relation. There are a few galaxies that do not follow this common TullyFisher law. Examples include the two gas-rich and dark matter dominated galaxies DDO 154 and NGC 2915 (Meurer et al. 1996): in these two galaxies, most of the baryons are in the extended HI disk component. DDO 154 and NGC 2915 lie about two magnitudes fainter than the usual $M_{B}$ - (velocity width) relation, but rise to it if the dominant $\mathrm{HI}$ component is notionally converted to stars with an $M / L$ ratio $\approx 1$. Their properties are summarised in Table 3 . Their stellar mass is about an order of magnitude less than their HI mass, which in turn is another order of magnitude less than the "total" dynamical mass. The last row of the table shows that the ratio of total mass to baryonic mass is again similar to that for the Milky Way $(\sim 20)$. The observation that these HI-dominated galaxies DDO 154 and NGC 2915 lie on the Tully-Fisher relation when their HI mass is notionally transformed to light is a further indication that 
the dark/baryon mass ratio is roughly constant from galaxy to galaxy, even for these extreme systems. We can regard these very extended HI disks as zero surface brightness (ZSB) disks.

IB consider the baryon contribution from LSB galaxies. The apparently flat number distribution of surface densities suggests that the baryon density $\Omega_{b}$ lies in the range $0.014<\Omega_{b}<0.025$. The likely nucleosynthesis bounds on $\Omega_{b}$ are $0.02<\Omega_{b}<0.03$. The volume-corrected estimate of the $\Omega_{b}$ contribution from luminous matter is about 0.003 (Persic and Salucci 1992). So it is possible that a substantial fraction of the baryons could lie in LSBG. However Briggs (1997) finds that LSBG with $M>10^{7} M_{\odot}$ contribute $<10 \%$ to the local HI density, and probably also little to the total mass and luminosity density.

\section{LSBG as systems of high angular momentum}

The angular momentum of aggregating systems in the early universe is probably tidally acquired. Simulations show that the parameter $\lambda=J|E|^{1 / 2} G^{-1} M^{-5 / 2}$ for halos lies in the range 0 to 0.15 , with mean value 0.05 ( $J$ is the angular momentum, $E$ the binding energy and $M$ the mass). Assume that gas and dark matter are initially well mixed with the same specific angular momentum $J / M$, and are truncated at a radius $r_{t}$. The gas dissipates to form an equilibrium exponential disk with scale length $h$ in the potential of the halo. Then the collapse factor $r_{t} / h=\sqrt{2} / \lambda \approx 30$ (Fall and Efstathiou 1980). For example, for the Milky Way, $h \approx 4 \mathrm{kpc}$ so $r_{t} \approx 120 \mathrm{kpc}$ which is consistent with the M31 timing estimates. High- $\lambda$ galaxies have longer scale lengths and lower surface densities. For example, increasing $\lambda$ from 0.05 to 0.15 increases $h$ by a factor 3 in the mean and reduces the surface density by about a factor 10 . This is consistent with the larger scale lengths observed for LBSG by Zwaan et al. (1995).

Dalcanton et al. (1997) and Jiminez et al. (1998) made more detailed models of this kind. Dalcanton et al. adopt a probability distribution of $\lambda$ from simulations, take a Schechter-like distribution of masses, include baryonic compression and predict the distribution of disk galaxies in the $\left(\mu_{o}, h\right)$ plane. Comparing their expected distribution with the observed distribution suggests that many galaxies remain to be discovered in some regions of this plane.

\section{LSBG in Clusters of Galaxies}

The cluster environment is often regarded as hostile to the formation and survival of LSBG. However the harrassment process (Moore et al. 1996) may contribute to the production of LSBG in clusters. Although LSBG in clusters are interesting for understanding the baryon content of clusters, they may tell us more about the dynamics of the cluster environment than about the nature of the LSBG phenomenon. We know from planetary nebula studies that a significant fraction of the stellar mass of the Virgo cluster lies in the intracluster medium (e.g. Mendez et al. 1997, Feldmeier et al. 1998). These intracluster stars are probably harrassment debris. The bimodality of the K-band distribution of central surface brightness for disk galaxies in the UMa cluster, discovered by Tully and Verheijen (1997), may be another possible dynamical effect of the cluster environment, although the long crossing time $\left(\sim 0.5 H_{\circ}^{-1}\right)$ may argue against 
this interpretation. It would be interesting to know if the UMa cluster has an intracluster stellar debris population like that of the Virgo cluster.

\section{HIPASS and LSBG}

The HIPASS survey (see Webster's paper in this volume) covers about 27,000 square degrees of sky and will identify gas-rich galaxies independent of their optical properties. It will give an optically unbiased view of the distribution of galaxies in the (surface brightness - luminosity) plane. The HIPASS survey has its own selection effects. For galaxies that overfill the Parkes beam (i.e. galaxies with diameter in $\mathrm{kpc}>4.4 \times$ distance in $\mathrm{Mpc}$ ), the surface density limit is $N_{H I}>5 \times 10^{17} \Delta V^{1 / 2} \mathrm{~cm}^{-2}$, where $\Delta V$ is the velocity width of the HI profile in $\mathrm{km} \mathrm{s}^{-1}$. For smaller galaxies, the HI mass limit is $M_{H I}>5 \times 10^{4} D^{2} \Delta V^{1 / 2} M_{\odot}$, where $D$ is the distance in Mpc. For example, for $D=10 \mathrm{Mpc}$ and $\Delta V=100$ $\mathrm{km} \mathrm{s}^{-1}, M_{H I}>5 \times 10^{7} M_{\odot}$. Objects like NGC 2915 can be detected out to 50 Mpc. Galaxies with $\Delta V=150 \mathrm{~km} \mathrm{~s}^{-1}$ that fill the Parkes beam can be detected down to surface density levels of $6 \times 10^{18} \mathrm{~cm}^{-2}$.

Despite the opportunities that HIPASS offers, we should remember the results of Zwaan et al. (1997) from their study of the Arecibo strip: 61 detections in 65 square degrees down to a limiting $N_{H I} \approx 10^{18} \mathrm{~cm}^{-2}$. They found that:

- The HI mass function for galaxies down to $M_{H I}=10^{7} M_{\odot}$ is similar to the HI mass function for optical galaxies.

- There does not appear to be a large class of gas-rich dwarfs or LSBG that were not previously detected optically. The HI content is dominated by high-mass galaxies with $M \sim 10^{9}-10^{10} M_{\odot}$.

- The lower limit to the average HI surface density is about $10^{19.7} \mathrm{~cm}^{-2}$, despite the sensitivity of the survey to much lower surface densities. Is this limit due to ionization of lower column density systems or is it more fundamental ?

\section{References}

Bothun, G., Impey, C., McGaugh, S. 1997, PASP, 109, 745

Briggs, F. 1997, ApJ, 484, 618

Dalcanton, J., Spergel, D., Summers, F. 1997, ApJ, 482, 659

de Blok, W.J.G., McGaugh, S. 1997, MNRAS, 290, 533

Fall, S.M., Efstathiou, G. 1980, MNRAS, 193, 189

Feldmeier, J., Ciardullo, R., Jacoby, G. 1998, ApJ, 503, 109

Freeman, K.C. 1970, ApJ, 160, 811

Freeman, K.C. 1996, in "Unsolved Problems of the Milky Way" (IAU Symposium 169), ed L. Blitz \& P. Teuben (Kluwer), 645

Freeman, K.C. 1997, Proc.Astron.Soc.Austr., 14, 4

Impey, C., Bothun, G. 1997, ARA\&A, 35, 267 
Jiminez, R., Padoan, P., Matteuci, F., Heavens, A. 1998, MNRAS, 299, 123

Meurer, G.M., Carignan, C., Beaulieu, S.F., Freeman, K.C. 1996, AJ, 111, 1551 Mendez, R.H., Guerrero, M.Ai, Freeman, K.C., Arnaboldi, M., Kudritzki, R.P., Hopp, U., Capaccioli, M., Ford, H. 1997, ApJ, 491, L23

Moore, B., Katz, N., Lake, G., Dressler, A., Oemler, A. 1996, Nature, 379, 613 Persic, M., Salucci, P. 1992, MNRAS, 258, 14P

Tully, B., Verheijen, M. 1997, ApJ, 484, 145

Zwaan, M.A., van der Hulst, J.M., de Blok, W.J.G., McGaugh, S.S. 1995, MNRAS, 273, 35P

Zwaan, M.A., Briggs, F.H., Sprayberry, D., Sorar, E. 1997, ApJ, 490, 173 